\title{
BMJ Open Associations between gastro-oesophageal reflux disease and a range of diseases: an umbrella review of systematic reviews and meta-analyses
}

\author{
JinJing Tan, ${ }^{1,2}$ Liqun Li ${ }^{3}$ Xiaoyan Huang, ${ }^{3}$ Chengning Yang, ${ }^{3}$ Xue Liang, ${ }^{3}$ \\ Yina Zhao, ${ }^{3}$ Jieru Xie, ${ }^{4}$ Ran Chen, ${ }^{3}$ Daogang Wang, ${ }^{3}$ Sheng Xie (1) ${ }^{2}$
}

To cite: Tan J, Li L, Huang X, et al. Associations between gastro-oesophageal reflux disease and a range of diseases: an umbrella review of systematic reviews and meta-analyses. BMJ Open 2020;10:e038450. doi:10.1136/ bmjopen-2020-038450

- Prepublication history and additional material for this paper are available online. To view these files, please visit the journal online (http://dx.doi org/10.1136/bmjopen-2020038450).

JT, LL, XH and CY contributed equally.

Received 13 March 2020 Revised 13 November 2020 Accepted 19 November 2020

Check for updates

(C) Author(s) (or their employer(s)) 2020. Re-use permitted under CC BY-NC. No commercial re-use. See rights and permissions. Published by BMJ.

For numbered affiliations see end of article.

Correspondence to

Dr Sheng Xie;

xiesheng2018touga0@163.com

\section{ABSTRACT}

Objective Numerous meta-analyses have revealed the association between gastro-oesophageal reflux disease (GORD) and a range of diseases; however, the certainty of the evidence remains unclear. This study aimed to summarise and assess the certainty of evidence derived from meta-analyses.

Methods Embase, PubMed, Web of Science, Cochrane Databases of Systematic Reviews, CNKI and Wangfang databases from their inception to 22 February 2020 were queried for systematic reviews and meta-analyses on the association between GORD and various diseases. The methodological quality of the included studies was assessed using A Measurement Tool to Assess Systematic Reviews 2 (AMSTAR 2), and evidence certainty was evaluated using the Grading of Recommendations, Assessment, Development, and Evaluation (GRADE) system. Statistical analysis was conducted using Stata V.15.

Results Ten publications with associations between GORD and different types of diseases were included. There was high heterogeneity $\left(I^{2}>75 \%\right)$ among seven independent meta-analyses. Evidence for publication bias in two independent meta-analyses was also observed. According to the AMSTAR 2 approach, the methodological quality was high for $20 \%$ of meta-analyses, moderate for $10 \%$, low for $40 \%$ and critically low for $30 \%$. Based on GRADE approach, the certainty of evidence was high for the association between GORD and higher risk of chronic obstructive pulmonary disease (COPD) exacerbation (OR $5.37 ; 95 \% \mathrm{Cl} 2.71$ to 10.64 ) and higher prevalence of oesophageal adenocarcinoma (OR 4.57; $95 \% \mathrm{Cl} 3.89$ to 5.36), and it was moderate for the association between GORD and higher chronic rhinosinusitis prevalence (OR 2.16; 95\% Cl 1.37 to 3.48).

Conclusion The association between GORD and a range of diseases was extensively studied, and our findings revealed a high certainty of evidence of the association between GORD and an increased risk of COPD exacerbation as well as increased prevalence of oesophageal adenocarcinoma. Further investigations using systematic reviews and meta-analyses of high methodological quality that include prospective large cohort studies and adjusted confounders are warranted. PROSPERO registration number CRD42019122264.
Strengths and limitations of this study

- This umbrella review is the first synthesis of systematic reviews and meta-analyses to consider the association between gastro-oesophageal reflux disease (GORD) and diverse diseases.

- These results provide insights into the association between GORD and diverse diseases.

- The associations between GORD and most of diseases observed in this umbrella review may reflect uncertainty.

- Evidence from original observational studies was not discussed in this study, which may result in conclusion bias.

\section{INTRODUCTION}

Gastro-oesophageal reflux disease (GORD) is a condition characterised by stomach content reflux causing discomfort and other symptoms. ${ }^{1}$ The prevalence of GORD is increasing worldwide. ${ }^{2}$ The oesophagus is located within the chest area and connects the throat and stomach cavities with the neighbouring nondigestive tract organs, namely the larynx, heart and respiratory tract. Reflux of stomach contents (eg, pepsin, gastric acid or bile) into the oesophagus may damage the specialised physiological structure of neighbouring organs. Therefore, GORD is a risk factor for multiple diseases.

The association between GORD and various diseases has been extensively investigated, and studies have revealed that GORD is associated with respiratory diseases (eg, chronic obstructive pulmonary disease (COPD) $),{ }^{3}$ cardiovascular diseases (eg, coronary heart disease and atrial fibrillation), ${ }^{45}$ mental disorders, ${ }^{6}$ head and neck diseases (eg, chronic rhinosinusitis (CRS) $)^{7}$ and cancer (eg, laryngeal cancer). ${ }^{8}$ These findings suggest that GORD threatens physical health as well as causing various diseases that further aggravate 
the economical and psychological burden of patients. ${ }^{36}$ Recognising the associations between GORD and various diseases may be important for public health prevention based on the substantial global burden of diseases. However, to our knowledge, no study has comprehensively summarised these reported associations. Therefore, this study summarises the scope of associations between GORD and diverse diseases.

Although many systematic reviews and meta-analyses have shown the association between GORD and various diseases, the certainty of the evidence remains unclear. The risk of bias, scheme design defects, publication bias or inconsistencies in meta-analysis studies may decrease the certainty of evidence. It is important to clarify the certainty of the associations between GORD and the risk of various diseases for diagnosis and treatment. Therefore, the scope of the associations between GORD and diverse diseases needs to be summarised, and the strength and validity regarding these associations should be clarified using validated tools. An umbrella review is a useful tool to systematically search, collect and assess the existing evidence derived from various systematic reviews and meta-analyses on any clinical health outcomes related to a particular exposure. ${ }^{9}{ }^{10}$ It provides an overview of the range and validity of the reported associations.

This study aimed to perform an umbrella review of meta-analyses on the association between GORD and various diseases and to assess the strength and validity of such evidence.

\section{MATERIALS AND METHODS}

The presentation of this umbrella review was in accordance with the Preferred Reporting Items for Systematic Reviews and Meta-Analyses statement. ${ }^{11}$ This umbrella review protocol has been registered in PROSPERO as CRD42019122264 (https://www.crd.york.ac.uk/ prospero/).

\section{Literature search}

Two researchers (YZ and XL) independently and comprehensively performed computerised searches on Embase, PubMed, Web of Science, CNKI, the Wangfang database and the Cochrane Database of Systematic Reviews to identify systematic reviews and meta-analyses of observational studies on the associations between GORD and diverse diseases. The searched timeframe was from database inception to 22 February 2020. The search strategy involved a combination of medical subject terms, words and free text. The search algorithms employed for each database can be found in online supplemental appendix 1. References from relevant reviews and meta-analyses were also manually screened. All identified publications were managed using EndNote. The two reviewers (LL and JT) independently screened the titles, abstracts and full texts of eligible articles in accordance with the inclusion and exclusion criteria. Any discrepancy was resolved via discussion, and a third researcher (SX) arbitrated all discrepancies that could not be resolved through discussion.

\section{Eligibility and exclusion criteria}

Studies were included if they met the following criteria: (1) included systematic reviews and meta-analyses of observational studies (any type of observational study design, eg, case-control study and cross-sectional study) with pooled summary effects (eg, relative risks (RRs), ORs and their 95\% CIs); (2) considered GORD as exposure; (3) included studies in which GORD was diagnosed via validated methods (eg, oesophagogastroduodenoscopy and 24-hour $\mathrm{pH}$ monitoring); and (4) considered the associations (ie, prevalence, incidence or risk) between GORD and diverse diseases (eg, cancer, respiratory disease and digestive disease) as the outcome.

Studies were excluded if they were systematic reviews without meta-analyses. Animal and laboratory studies were also excluded. When two or more meta-analyses were performed for the same review question, the metaanalysis with the largest sample size and the latest date of publication for each outcome was selected to avoid duplicate studies. If the most current meta-analysis was not the one with the largest sample size, then the reason for this discrepancy was explored. In our review, the meta-analysis with the largest sample size was included.

\section{Data extraction}

Data from each eligible systematic review and metaanalyses were independently extracted by two investigators (LL and CY). The abstracted information was carefully checked by a third investigator (XH). The name of the first author, publication year, outcomes examined, number of cases and participants, number of included studies, study design of the primary study, method of GORD diagnosis and risk of bias assessment tool were extracted using a predesigned data extraction form. For each eligible meta-analysis, the reported pooled summary effects (ORs and their 95\% CIs) and their p values as well as results of subgroup analyses were extracted. Simultaneously, we extracted the $\mathrm{I}^{2}$ and $\mathrm{p}$ values of Cochran's $\mathrm{Q}$ test to evaluate the heterogeneity between meta-analysis studies. The results of publication bias and $p$ values of Egger's test were extracted to determine whether publication bias existed in the meta-analysis. If two or more outcomes of interest were examined in a systematic review and meta-analysis, each outcome was recorded separately.

\section{Assessment of methodological quality}

The methodological quality of the included studies was independently assessed by two investigators (DW and CY) using AMSTAR2, a measurement tool used to assess the methodological quality of systematic reviews, ${ }^{12}$ and the results were checked by a third investigator $(\mathrm{XH})$. Inconsistencies were resolved by discussion. AMSTAR 2 contains 16 checklists (seven critical and nine noncritical checklists) for assessing the quality of systematic reviews and meta-analyses. According to AMSTAR 2, 
items $2,4,7,9,11,13$ and 15 were defined as critical domains, and items $1,3,5,6,8,10,12,14$ and 16 were defined as noncritical domains. The rating criteria of the methodological quality of meta-analyses were as follows: high quality when one or no noncritical weakness was found, moderate quality when two or more non-critical weaknesses were found, low quality when one critical flaw with or without non-critical weaknesses was found and critically low quality when two or more critical flaws with or without noncritical weaknesses were found.

\section{Assessment of certainty evidence}

In this umbrella review, the GRADE system was used to evaluate the certainties of evidence derived from systematic reviews and meta-analyses of observational studies on the associations between GORD and various diseases. ${ }^{1314}$ This system includes five factors (risk of bias, inconsistency, indirectness, imprecision and publication bias) for downgrading the certainty of evidence and three factors (large effect, dose response and plausible residual confounding) for upgrading the certainty of evidence. When there were serious or very serious downgrading factors, the certainty of the evidence was lower by one or two levels, respectively. If the effect was large (OR either $>2.0$ or $<0.5$ ) or very large (OR either $>5.0$ or $<0.2$ ), then evidence quality was upgraded by one or two levels, respectively. If there was evidence that the influence of all plausible confounding would reduce a demonstrated effect or suggest a spurious effect, then evidence quality was upgraded by one level. The rating criteria of the certainty of evidence were as follows: (1) the certainty of the primary evidence of an observational study was considered 'low'; (2) the certainty of evidence was classified 'very low' by downgrading one level; (3) the certainty of evidence was rated 'moderate' by upgrading one level; and (4) the certainty of evidence was graded 'high' by upgrading two levels. GRADE assessment was conducted by two researchers (LL and JT), and the results were checked by a third investigator (XH). Inconsistencies were resolved by discussion.

\section{Data analysis}

We recalculated the summary ORs and corresponding 95\% CIs. A random-effects model was used for calculation when $\mathrm{I}^{2}>50 \%$ was observed; otherwise, a fixed-effects model was employed. The heterogeneity among different studies was assessed using the $\mathrm{I}^{2}$ and the $\mathrm{p}$ value results of Cochran's Q test. Publication bias was estimated using Egger's test. Differences for which $\mathrm{p}<0.1$ were deemed statistically significant. No heterogeneity, low heterogeneity, moderate heterogeneity and high heterogeneity were indicated by an $\mathrm{I}^{2}$ of $\leq 25 \%, 25 \%-50 \%, 50 \%-75 \%$ and $\geq 75 \%$, respectively. We also conducted subgroup analysis to explain the sources of heterogeneity. Statistical analyses were conducted using Stata V.15. Figures were prepared using GraphPad Prism V.5.

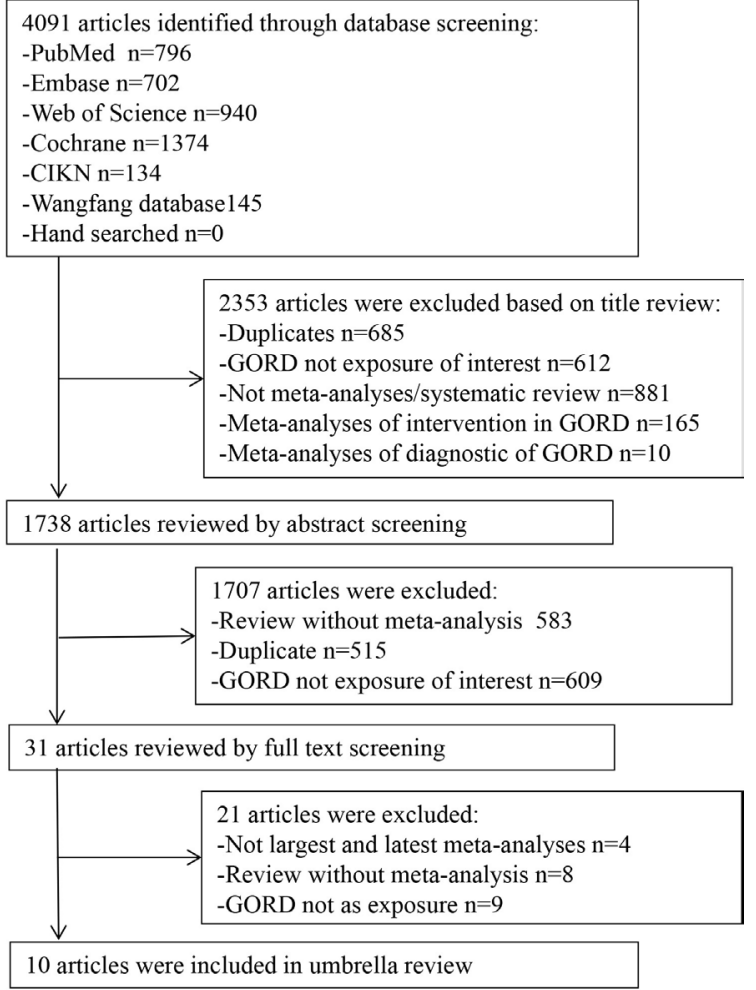

Figure 1: Flowchart of the study selection process for umbrella review

Figure 1 Flow chart of study selection process for umbrella review. GORD, gastro-oesophageal reflux disease.

\section{Patient and public involvement}

Patients and the public were not involved in the design of this study, conducting, reporting or dissemination of plans of our research.

\section{RESULTS}

\section{Description of the meta-analyses}

In summary, 4091 articles meeting our search criteria were initially identified from six databases. Twenty-one articles ${ }^{15-35}$ were excluded after reviewing by full text and were shown in online supplemental table S1. Ten articles $^{36-45}$ published between 2007 and 2020 were selected (figure 1). The characteristics of the eligible meta-analyses are summarised in table 1 . A total of 92 individual studies, consisting of 14 cross-sectional studies and 78 case-controlled studies, were included. The study estimates pooled per meta-analysis ranged from 2 to 26 , and the median of the study estimate was 6 . Three metaanalyses $^{3640} 42$ included more than 10 primary studies. The number of cases ranged from 82 to 36503 , and the number of participants ranged from 198 to 254978 in the meta-analyses. Associations between GORD and idiopathic pulmonary fibrosis (IPF), ${ }^{36}$ asthma, ${ }^{37} \mathrm{COPD}$ exacerbation, ${ }^{38}$ CRS,${ }^{39}$ laryngeal malignancy, ${ }^{40}$ oesophageal adenocarcinoma (OAC) ${ }^{41}$ Barrett's oesophagus, ${ }^{42}$ nonalcoholic fatty liver disease (NAFLD) ${ }^{43}$ obstructive sleep apnea-hypopnoea syndrome (OSAHS) ${ }^{44}$ and pharyngeal cancer ${ }^{45}$ were found. 


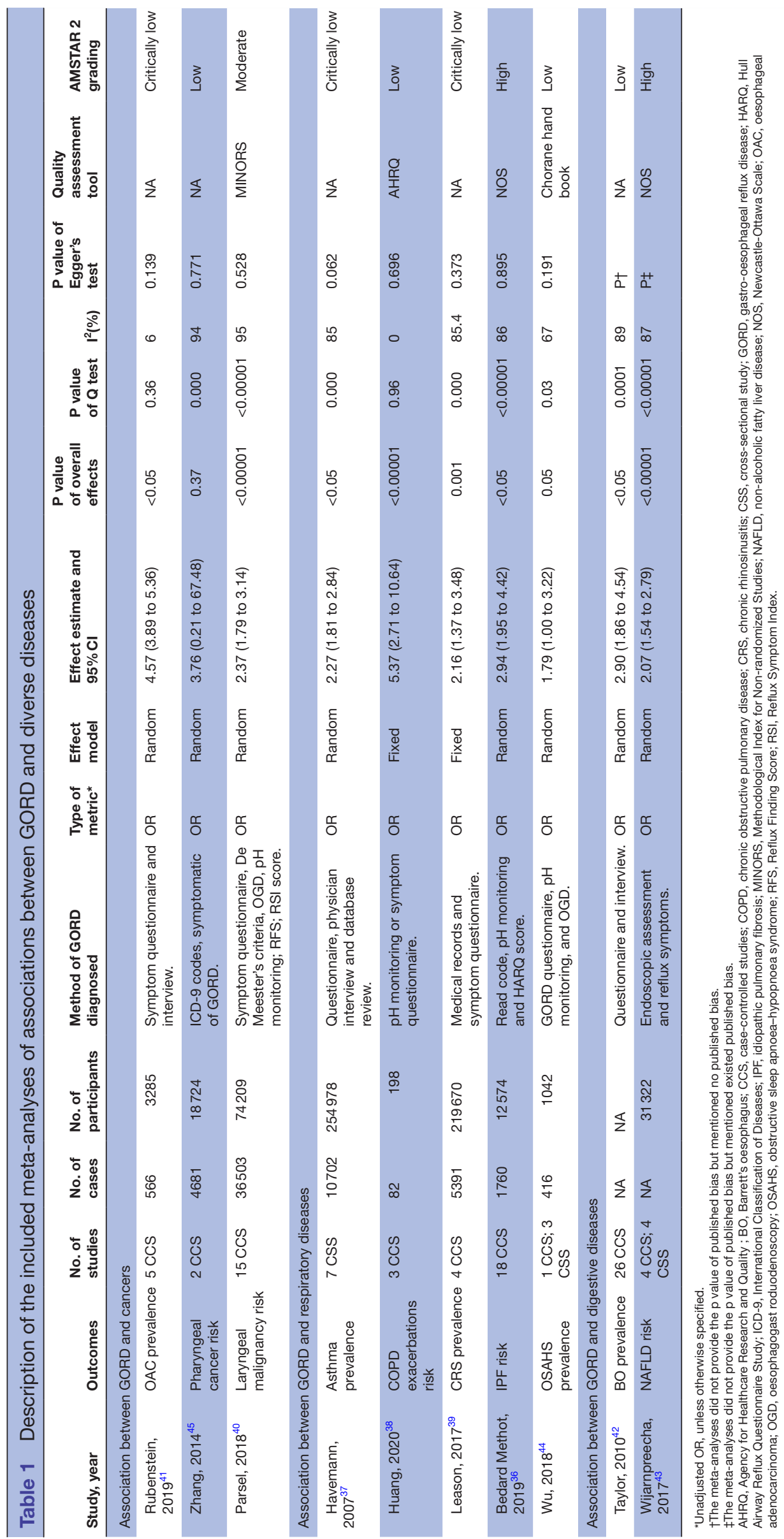




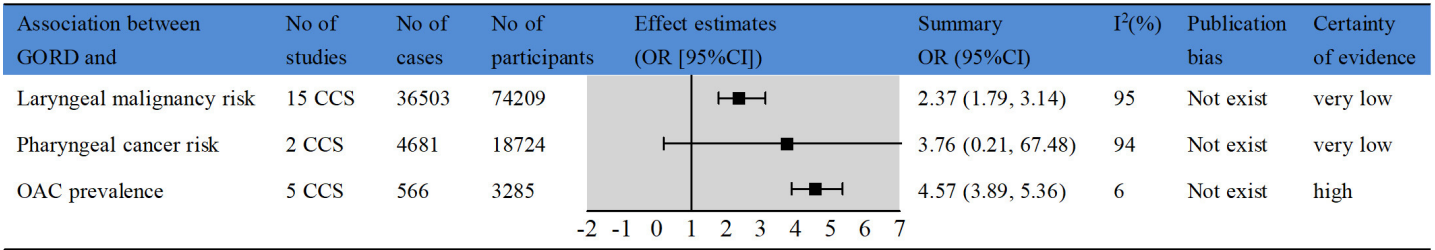

Figure 2 Summary ORs with 95\% Cls and certainty of evidence for association between GORD and cancers. Data are based on results from 3 published meta-analyses. CSS, Case controlled study; OAC, oesophageal adenocarcinoma; GORD, gastrooesophageal reflux disease.

\section{Summary of the methodological quality of the included meta- analyses}

The methodological qualities of the included meta-analyses were assessed using AMSTAR 2, and the detailed results are shown in online supplemental table S2. Five (40\%) metaanalyses did not report explicit statements or protocols. Five $(40 \%)$ meta-analyses did not use comprehensive literature search strategies. Six $(50 \%)$ of the meta-analyses did not assess the risk of bias in the primary studies. None of the meta-analyses reported the details of funding sources for the included studies. Overall, the methodological qualities of the included meta-analyses were categorised as high for $20 \%(n=2)$, moderate for $10 \%(n=1)$, low for $40 \%(n=4)$ and critically low for $30 \%(n=3)$ (table 1$)$.

\section{Certainty of evidence of the associations between GORD and diverse diseases}

A total of 10 associations between GORD and different types of diseases were found: cancer $(n=3)$, respiratory diseases $(n=5)$ and digestive diseases $(n=2)$. Nine of these associations had nominal significance. The detailed results of the certainty of evidence are summarised in online supplemental table S3. Overall, the certainties of evidence were graded high, moderate, low and very low for $20 \% \quad(n=2)$, $10 \%(\mathrm{n}=1), 10 \%(\mathrm{n}=1)$ and $60 \%(\mathrm{n}=6)$ of the associations, respectively (figures $2-4$ ).

\section{The associations between GORD and cancers}

Figure 2 shows that the individuals with weekly GORD symptoms have a fivefold increase in the odds of developing OAC ( $\mathrm{OR}=4.57 ; 95 \%$ CI 3.89 to 5.36), and the certainty of evidence was rated as high. ${ }^{41}$ A very low certainty of evidence indicated that GORD is associated with an increased risk of laryngeal malignancy by more than twofold ( $\mathrm{OR}=2.37 ; 95 \% \mathrm{CI}$ 1.79 to 3.14$)^{40}$ (figure 2 ). However, a very low certainty of evidence showed that there was no significant association between GORD and pharyngeal cancer. ${ }^{45}$ The results of subgroup analyses are shown in table 2 . The individuals with daily GORD symptoms had a sevenfold increase in odds of developing OAC. By stratifying based on duration of GORD symptoms, the odds of developing OAC were higher among individuals with GORD symptoms of at least 20 years was higher among individuals with GORD symptoms of less than 10-15 years. Based on ethnicity, GORD increased the risk of laryngeal malignancy in Europeans, Asians and Americans, particularly in Asians. GORD significantly increased the prevalence of OAC in Europeans, Australians and Americans. Additionally, GORD increased the risk of laryngeal malignancy by approximately fourfold based on diagnoses of GORD using objective methods. However, there was no association between GORD and laryngeal malignancy based on diagnoses of GORD using subjective methods.

\section{The associations between GORD and respiratory diseases}

Figure 3 shows the association between GORD and increased risk of COPD exacerbation ( $\mathrm{OR}=5.37 ; 95 \%$ CI 2.71 to 10.64$)$, and the certainty of evidence was high. ${ }^{38}$ The asthma prevalence in individuals with GORD was higher than those without GORD (OR=2.27; 95\% CI 1.81 to 2.84$)$, but the certainty of evidence was very low. ${ }^{37}$ CRS prevalence increased by more than twofold in patients with GORD (OR=2.16; 95\% CI 1.37 to 3.48 ), and the certainty of evidence was moderate. ${ }^{39}$ The summary OR for the association between GORD and IPF was OR=2.94 (95\% CI 1.95 to 4.42$)$, but the certainty of evidence was very low. ${ }^{36}$ The certainty of the positive association between GORD and OSAHS (OR=1.79; 95\% CI 1.00 to 3.22) was very low. ${ }^{44}$

The results of subgroup analyses are shown in table 2. GORD was associated with an increase in the risk of OSAHS

\begin{tabular}{|c|c|c|c|c|c|c|c|c|}
\hline $\begin{array}{l}\text { Association between } \\
\text { GORD and }\end{array}$ & No of studies & $\begin{array}{l}\text { No of } \\
\text { cases }\end{array}$ & $\begin{array}{l}\text { No of } \\
\text { participants }\end{array}$ & $\begin{array}{l}\text { Effect estimates } \\
(\text { OR }[95 \% \mathrm{CI}])\end{array}$ & $\begin{array}{l}\text { Summary } \\
\text { OR }(95 \% \mathrm{CI})\end{array}$ & $I^{2}(\%)$ & $\begin{array}{l}\text { Publication } \\
\text { bias }\end{array}$ & $\begin{array}{l}\text { Certainty of } \\
\text { evidence }\end{array}$ \\
\hline CRS prevalence & $4 \mathrm{CCS}$ & 5391 & 219670 & $1+-1$ & $2.16(1.37,3.48)$ & 85.4 & not exist & moderate \\
\hline OSAHS prevalence & $1 \mathrm{CCS} ; 3 \mathrm{CSS}$ & 416 & 1042 & -1 & $1.79(1.00,3.22)$ & 67 & not exist & very low \\
\hline IPF risk & $18 \mathrm{CCS}$ & 1760 & 12574 & $\mapsto$ & $2.94(1.95,4.42)$ & 86 & not exist & very low \\
\hline Asthma prevalence & $7 \mathrm{CSS}$ & 10702 & 254978 & $\mathrm{HPH}$ & $2.27(1.81,2.84)$ & 85 & exist & very low \\
\hline COPD exacerbations risk & $3 \mathrm{CCS}$ & 82 & 198 & $\longmapsto$ & $5.37(2.71,10.64)$ & 0 & not exist & high \\
\hline
\end{tabular}

Figure 3 Summary ORs with 95\% Cls and certainty of evidence for association between GORD and respiratory diseases. Data are based on results from five published meta-analyses. CCS, case-controlled study; COPD, chronic obstructive pulmonary disease; CRS, chronic rhinosinusitis; CSS, case-sectional study; GORD, gastro-oesophageal reflux disease; OIPF, idiapathic pulmonary fibrosis; OSAHS, obstructive sleep apnoea-hypopnoea syndrome. 


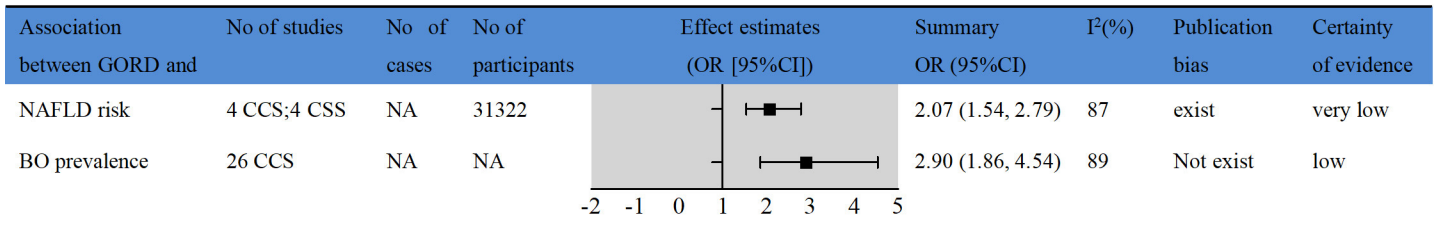

Figure 4 Summary ORs with $95 \% \mathrm{Cls}$ and certainty of evidence for association between GORD and digestive diseases. Data are based on results from two published meta-analyses. BO, Barrett's oesophagus; CCS, case-controlled study; CSS, casesectional study; GORD, gastro-oesophageal reflux disease; NA, not available; NAFLD, non-alcoholic fatty liver disease.

based on the diagnoses of GORD using objective methods. However, there was no association between GORD and OSAHS based on the diagnosis of GORD using subjective methods. Based on the age of the participants, GORD was associated with a higher risk of CRS in both adults and children, particularly in children ( $\mathrm{OR}=3.20 ; 95 \%$ CI 2.39 to 4.27$)$. Based on ethnicity, GORD was associated with an increase in the risk of asthma in Europeans, Asians and Americans, particularly in Asians, with a summary OR of 5.56. GORD was associated with a higher prevalence of OSAHS in Americans, but not in Asians, and GORD was associated with an increase in the risk of IPF in Europeans, Asians, Americans and Africans, but not in Canadians.

\section{The associations between GORD and digestive diseases}

The prevalence of Barrett's oesophagus in individuals with GORD was approximately three times higher in those without GORD ( $\mathrm{OR}=2.90$; 95\% CI 1.86 to 4.54$)$, and the certainty of evidence was rated as $\operatorname{low}^{42}$ (figure 4 ). Based on ethnicity, GORD was associated with an increase in the prevalence of Barrett's oesophagus in Americans by twofold. However, there were no associations between GORD and Barrett's oesophagus based on studies involving Asians $(\mathrm{OR}=1.62 ; 95 \%$ CI 0.813 to 3.24$)$ and Europeans $(\mathrm{OR}=3.00$; $95 \%$ CI 0.901 to 9.99 ) (table 2). GORD was significantly associated with an increase in the risk of NAFLD with a very low certainty of evidence $^{43}$ (figure 4).

\section{Heterogeneity and publication bias of the included meta- analyses}

The heterogeneity and publication bias of the included meta-analyses are summarised in table 1 . Eight of the included systematic reviews with meta-analyses showed heterogeneity $\left(\mathrm{I}^{2} \geq 30 \%\right)$. Among these, seven showed high heterogeneity with an $\mathrm{I}^{2}$ of $>75 \%,{ }^{36} 373940424345$ and one meta-analysis showed moderate heterogeneity with an $\mathrm{I}^{2}$ of $67 \%{ }^{44}$ The results of subgroup analyses showed that heterogeneity in studies significantly decreased when stratifying by age of participants, ${ }^{39}$ study design, ${ }^{43}$ ethnicity ${ }^{3637} 40-4244$ and diagnostic methods. ${ }^{44}$ Two of the included meta-analyses showed statistical evidence of publication bias. ${ }^{373}$

\section{DISCUSSION}

\section{Principal findings}

The influence of GORD on the development of diverse diseases has been examined in many published metaanalyses. This umbrella review included 10 published meta-analyses that investigated on the association between GORD and 10 types of diseases. Approximately $80 \%$ of the meta-analyses reported significant heterogeneity. Two meta-analyses showed statistically significant evidence of publication bias. The methodological qualities were categorised as high only for two meta-analyses and moderate for one. The certainties of evidence were graded as high for the association between GORD and increased risk of COPD exacerbation and for the association between GORD and increased prevalence of OAC.

\section{Comparison with other studies}

This umbrella review supported some of the recommendations as well as added related evidence. Recommendations on the contribution of GORD to the development of COPD were included in the guidelines. ${ }^{46}{ }^{47}$ This information was in accordance with our result that GORD was associated with an increased risk of COPD exacerbation. Therefore, controlling GORD in patients with COPD may contribute to the control of COPD and improve prognosis. Additionally, our findings showed that GORD was associated with an increase in the prevalence of OAC, indicating that GORD may play an important role in the aetiology of OAC, with a high certainty of evidence. ${ }^{41}$ This result indicates that controlling GORD might be beneficial in deterring OAC development. However, Australian guidelines state that the treatment of GORD with proton pump inhibitors (PPIs) does not influence OAC progression. ${ }^{48}$ Larger randomised controlled trials should be conducted in the future to confirm this discrepancy. Chinese guidelines have reported GORD as a risk factor of CRS but failed to establish the strength of their evidence. ${ }^{49}$ This umbrella review confirmed that the certainty of this evidence may be moderate.

Japanese guidelines recommended the management of the coexistence of a condition coexisting with GORD when treating asthma. ${ }^{50}$ If asthma is not been managed at any step during therapy, then the assessment of the coexisting of a condition with GORD is also recommended. ${ }^{51}$ Korean guidelines indicate that GORD occurs in up to $87 \%$ of patients with IPF and can contribute to the exacerbation of IPF. ${ }^{52}$ The American College of Gastroenterology recommends the routine use of PPIs for Barrett's oesophagus even in the absence of symptoms. ${ }^{53}$ However, we found that the certainties of evidence for the association between GORD and these diseases are 'low' or 'very low', indicating that these associations should be assessed 
Table 2 The results of subgroup analysis of the included meta-analyses

\begin{tabular}{|c|c|c|c|c|c|c|}
\hline Subgroup classification & $\begin{array}{l}\text { Association between } \\
\text { GORD and }\end{array}$ & $\begin{array}{l}\text { Number of } \\
\text { studies }\end{array}$ & $\begin{array}{l}\text { Effect } \\
\text { model }\end{array}$ & OR $(95 \% \mathrm{Cl})$ & $\begin{array}{l}P \text { value of } \\
Q \text { test }\end{array}$ & $I^{2}(\%)$ \\
\hline \multicolumn{7}{|c|}{ Stratified by frequency of GORD symptoms } \\
\hline Daily symptoms & OAC prevalence ${ }^{41}$ & 5 CCS & $\mathrm{R}$ & 7.40 (4.94 to 11.1$)$ & 0.01 & 71 \\
\hline Weekly symptoms & OAC prevalence ${ }^{41}$ & $5 \operatorname{CCS}$ & $\mathrm{R}$ & 4.57 (3.89 to 5.36$)$ & 0.04 & 60 \\
\hline \multicolumn{7}{|c|}{ Stratified by duration of GORD symptoms } \\
\hline At least 20 years & OAC prevalence $e^{41}$ & 4 CCS & $\mathrm{R}$ & 5.41 (2.45 to 11.9$)$ & $<0.01$ & 89 \\
\hline Less than $10-15$ years & OAC prevalence ${ }^{41}$ & 4 CCS & $\mathrm{R}$ & 3.05 (1.53 to 6.08$)$ & $<0.01$ & 84 \\
\hline \multicolumn{7}{|c|}{ Stratified by diagnostic methods of GORD } \\
\hline \multirow[t]{3}{*}{ Subjective methods } & $\begin{array}{l}\text { Laryngeal malignancy } \\
\text { risk }^{40}\end{array}$ & 5 CCS & $\mathrm{F}$ & 1.43 (0.93 to 2.22$)$ & 0.1 & 48 \\
\hline & OSAHS prevalence ${ }^{41}$ & 2 CSS & $\mathrm{F}$ & 1.63 (1.21 to 2.19$)$ & 0.455 & 0 \\
\hline & IPF risk ${ }^{36}$ & $12 \operatorname{CCS}$ & $\mathrm{R}$ & 2.36 (1.82 to 3.05$)$ & 0 & 91.1 \\
\hline \multirow[t]{3}{*}{ Objective methods } & OSAHS prevalence ${ }^{44}$ & $1 \mathrm{Ccs}$ & NA & 1.21 (0.67 to 2.18 ) & NA & NA \\
\hline & IPF risk ${ }^{36}$ & $11 \mathrm{CCS}$ & $\mathrm{R}$ & 2.80 (1.57 to 5.00$)$ & 0.007 & 59 \\
\hline & $\begin{array}{l}\text { Laryngeal malignancy } \\
\text { risk }^{40}\end{array}$ & $11 \mathrm{CCS}$ & $\mathrm{F}$ & 3.82 (2.61 to 5.59 ) & 0.14 & 32 \\
\hline
\end{tabular}

Stratified by study design

\begin{tabular}{|c|c|c|c|c|c|c|}
\hline Prospective studies & $\begin{array}{l}\text { Laryngeal malignancy } \\
\text { risk }^{40}\end{array}$ & 12 CCS & $\mathrm{F}$ & 2.46 (1.57 to 3.85$)$ & NA & 68 \\
\hline Retrospective studies & $\begin{array}{l}\text { Laryngeal malignancy } \\
\text { risk }^{40}\end{array}$ & $8 \mathrm{CCS}$ & $\mathrm{F}$ & 2.68 (1.86 to 3.85$)$ & NA & 97 \\
\hline Cross-sectional studies & NAFLD risk ${ }^{43}$ & 4 CSS & $\mathrm{F}$ & 1.52 (1.15 to 2.00$)$ & 0.001 & 86 \\
\hline Case-control studies & NAFLD risk ${ }^{43}$ & $4 \mathrm{CCS}$ & $\mathrm{F}$ & 3.04 (2.27 to 4.06$)$ & 0.7 & 0 \\
\hline \multicolumn{7}{|c|}{ Stratified by age of participants } \\
\hline Adults with GORD & CRS prevalence ${ }^{39}$ & $3 \mathrm{CCS}$ & $\mathrm{F}$ & 1.66 (1.57 to 1.75$)$ & 0.426 & 0 \\
\hline Children with GORD & CRS prevalence ${ }^{39}$ & $1 \mathrm{CCS}$ & NA & 3.20 (2.39 to 4.27$)$ & NA & NA \\
\hline \multicolumn{7}{|c|}{ Stratified by ethnicity of participants } \\
\hline \multirow[t]{5}{*}{ Europeans } & Asthma prevalence $^{37}$ & $3 \mathrm{CSS}$ & $\mathrm{F}$ & 1.98 (1.79 to 2.20$)$ & 0.51 & 0 \\
\hline & BO prevalence ${ }^{42}$ & $8 \mathrm{CCS}$ & $\mathrm{R}$ & 3.00 (0.901 to 9.99$)$ & $<0.0001$ & 93 \\
\hline & OAC prevalence $e^{41}$ & $2 \operatorname{ccs}$ & $\mathrm{R}$ & 5.59 (3.02 to 10.33$)$ & 0.02 & 81 \\
\hline & IPF risk ${ }^{36}$ & $6 \mathrm{CCS}$ & $\mathrm{R}$ & 2.05 (1.69 to 2.49 ) & 0 & 77.5 \\
\hline & $\begin{array}{l}\text { Laryngeal malignancy } \\
\text { risk }^{40}\end{array}$ & $6 \mathrm{CCS}$ & $\mathrm{F}$ & 4.72 (3.16 to 7.06$)$ & 0.233 & 26.9 \\
\hline \multirow[t]{5}{*}{ Asians } & IPF risk ${ }^{36}$ & $2 \operatorname{CCS}$ & $\mathrm{F}$ & 4.28 (1.81 to 10.11$)$ & 0.548 & 0 \\
\hline & Asthma prevalence $^{37}$ & $2 \mathrm{css}$ & $\mathrm{R}$ & 5.56 (1.66 to 18.67$)$ & 0.005 & 87.5 \\
\hline & BO prevalence ${ }^{42}$ & $3 \mathrm{CCS}$ & $\mathrm{F}$ & $1.62(0.813$ to 3.24$)$ & 0.14 & 49 \\
\hline & $\begin{array}{l}\text { Laryngeal malignancy } \\
\text { risk }^{40}\end{array}$ & $2 \operatorname{ccs}$ & $\mathrm{F}$ & 4.79 (2.26 to 10.15$)$ & 0.78 & 0 \\
\hline & OSAHS prevalence ${ }^{44}$ & 2 CSS & $\mathrm{R}$ & $\begin{array}{l}8.88(0.15 \text { to } \\
528.17)\end{array}$ & 0.005 & 87.6 \\
\hline \multirow[t]{6}{*}{ Americans } & BO prevalence ${ }^{42}$ & $12 \operatorname{CCS}$ & $\mathrm{R}$ & 2.44 (1.42 to 4.23$)$ & $<0.0001$ & 87 \\
\hline & Asthma prevalence ${ }^{37}$ & 2 CSS & $\mathrm{F}$ & 1.69 (1.60 to 1.77$)$ & 0.64 & 0 \\
\hline & OAC prevalence $e^{41}$ & $3 \mathrm{CCS}$ & $\mathrm{F}$ & 4.09 (3.23 to 5.18$)$ & 0.87 & 0 \\
\hline & IPF risk ${ }^{36}$ & $8 \mathrm{CCS}$ & $\mathrm{R}$ & 3.27 (1.83 to 3.79$)$ & 0 & 89.2 \\
\hline & $\begin{array}{l}\text { Laryngeal malignancy } \\
\text { risk }^{40}\end{array}$ & $7 \operatorname{CCS}$ & $\mathrm{R}$ & 1.69 (1.21 to 2.36$)$ & 0 & 97.7 \\
\hline & OSAHS prevalence ${ }^{44}$ & $1 \mathrm{CCS} ; 1 \mathrm{CSS}$ & $\mathrm{F}$ & 1.53 (1.00 to 2.36$)$ & 0.25 & 25 \\
\hline
\end{tabular}


Table 2 Continued

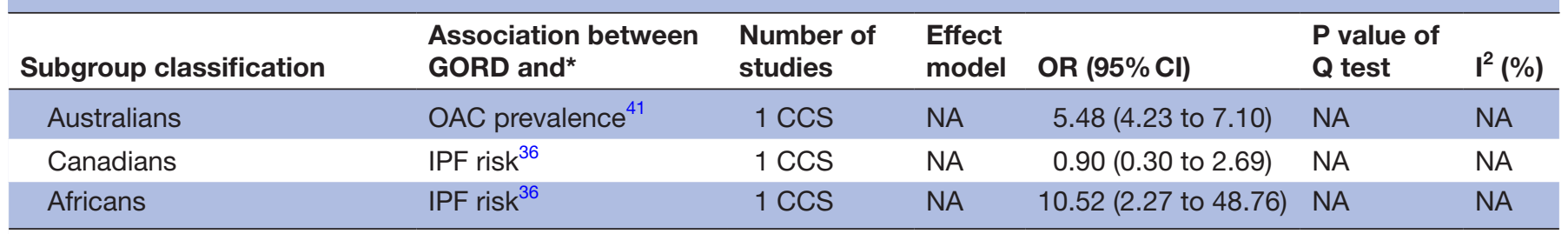

BO, Barrett's oesophagus; CCS, case-controlled studies; CRS, chronic rhinosinusitis; CSS, cross-sectional study; GORD, gastro-oesophageal reflux disease; IPF, idiopathic pulmonary fibrosis; NAFLD, non-alcoholic fatty liver disease; OAC, oesophageal adenocarcinoma; OSAHS, obstructive sleep apnoea-hypopnoea syndrome.

with caution. Although certainties of evidence for these associations were graded 'low' or 'very low', they might still provide ideas for clinicians. Therefore, more welldocumented research studies should be conducted to confirm these associations.

In a previous review, Chen ${ }^{54}$ summarised the findings of primary studies and meta-analyses on the association between GORD and non-digestive tract diseases. However, that review only focused on the association between GORD and non-digestive tract diseases and not that between GORD and digestive tract diseases. Furthermore, it was only a narrative review, not an umbrella review. The methodological quality of meta-analyses and the certainty of evidence of the existing evidence were not evaluated in this study. Our umbrella review not only provides a broad overview of the current existing evidence derived from meta-analyses but also evaluates the methodological quality of the published meta-analyses and the certainty of their evidence.

\section{Possible explanations}

We found that GORD was positively associated with an increased risk for nine diseases. However, all of the ORs abstracted from the included meta-analyses were not adjusted for confounders (eg, smoking and drinking). Nevertheless, the influence of confounding factors on the results cannot be ruled out, particularly in the analysis of respiratory diseases (eg, IPF and COPD exacerbation). Additionally, estimates with a very wide $95 \%$ CI, such as the association between GORD and pharyngeal cancer also affect the accuracy of evidence. Therefore, this association might be inconclusive.

High certainty of evidence was observed for the positive association between GORD and increased risk of COPD exacerbation. Microaspiration of gastric contents (eg, gastric acids, bile and pepsin) reflux may have an important role in the development of this disease. ${ }^{55} \mathrm{~A}$ previous study detected pepsin in the bronchoalveolar fluid of all of the patients with COPD ${ }^{56}$ Interestingly, pepsin levels in the lung could increase pulmonary protein oxidation. ${ }^{57}$ Additionally, the trigger of COPD exacerbations may be related to proximal acidic reflux, and extensive proximal acidic reflux may contribute to pulmonary oxidative stress. ${ }^{57} 58$ A previous study demonstrated an increase in protein oxidation in the lungs of patients with GORD compared with healthy individuals. ${ }^{57}$
Therefore, GORD may substantially contribute to inflammatory and oxidative damage in the lung. ${ }^{59}$ In addition, dysregulation of vagal nervous system induced by exposure of gastric acids is also a major mechanism explained the association between GORD and COPD exacerbation. ${ }^{60}$ Simultaneously, abnormal breathing physiology in patients promote the occurrence of GORD ${ }^{59}$ Both recurrent episodes of COPD and GORD require frequent use of medications. The frequent application of $\beta 2$-agonists could exacerbate gastrio-oesophageal reflux. This situation results in a pathological vicious cycle.

Additionally, a high certainty of evidence showed that GORD is associated with an increased prevalence of OAC, particularly in individuals with daily GORD symptoms and a symptom duration of at least 20 years. This association may be explained by biological changes caused by gastric content reflux. Gastric contents (eg, bile acids, pepsin and trypsin) promote interleukin-8 production from oesophageal epithelial cells that could induce epithelial cell transformation and promote cancer cell proliferation. ${ }^{6162}$ Additionally, a decrease in $\mathrm{pH}$ in the oesophagus plays an important role in the occurrence of cancer. ${ }^{63}$ In previous studies, oesophageal tissues are stimulated repeatedly by the refluxate, causing oesophageal tissues to be repeated damaged as well as inducing chronic inflammation, thereby promoting the development of cancer. ${ }^{6465}$

The association between GORD and increased prevalence of CRS warrants further investigation. The certainty of evidence was graded as moderate. A previous study established that pepsin may potentiate CRS by damaging mitochondria in nasal epithelial cells, which may explain the mechanism of GORD that is associated with an increase in CRS prevalence. ${ }^{66}$

Subgroup analyses showed that GORD increases the risk of Barrett's oesophagus in Americans, but not in Europeans and Asians. Differences in lifestyle and ethnicity might explain this difference. Associations without significance also should be interpreted with caution. Although GORDs were not associated with increased risk of Barrett's oesophagus in Europeans and Asians, these associations might also suggest an association between these two diseases because of the summary ORs of 3.00 and 1.62 , respectively. ${ }^{42}$

This study demonstrated that $80 \%$ of the metaanalyses had moderate to high heterogeneity $\left(\mathrm{I}^{2} \geq 50 \%\right)$. 
These sources of heterogeneity may be explained by differences in the age of participants ${ }^{39}$ study design ${ }^{43}$ ethnicity $^{36} 37$ 40-42 45 and diagnostic methods. ${ }^{40}$ High heterogeneity indicates that some associations may simply be false positives or exaggerated. ${ }^{6768}$ Therefore, considering the substantial heterogeneity, only the associations between GORD and higher risk of COPD exacerbations and OAC development are genuine, ${ }^{38}$ but this does not mean that other associations are not true. We found that two meta-analyses showed statistically significant evidence of publication bias. ${ }^{37} 43$ Publication bias favours the reporting of significant positive results. ${ }^{69}$ Therefore, the association between GORD and asthma and NAFLD should be interpreted with caution.

\section{Strengths, limitations and future studies}

This umbrella review has several strengths. It is the first to systematically summarise the associations between GORD and various diseases and to critically evaluate the methodological quality of meta-analyses and the certainty of existing evidence. The strength of an umbrella review has been demonstrated in other articles. ${ }^{10} 70$ In this review, AMSTAR 2 was used to assess the methodological quality of meta-analyses, ${ }^{12} 7172$ and the GRADE system ${ }^{14} 73$ was adopted to evaluate the certainty of evidence. AMSTAR 2 and GRADE are validated tools, and their efficiency and reliability have been exhibited in other published studies. ${ }^{74-76}$ Some defects of the existing published metaanalyses were found throughout this umbrella review, which may facilitate the improvement of future studies. Our umbrella review also found gaps in evidence of the associations between GORD and other diseases (eg, rheumatoid arthritis), which should be considered in future studies.

This umbrella review also has several limitations. Although AMSTAR 2 and GRADE are validated tools, the use of other tools such as Confidence in the Evidence from Reviews of Qualitative Research and Risk Of Bias In Systematic reviews could have led to different conclusions regarding the certainty of evidence. We only focused on existing and published systematic reviews with meta-analyses on observational studies, which may have resulted in conclusion bias. Evidence from individual observational studies was beyond the scope of our discussion. ${ }^{45}$ We did not use $\tau^{2}$ and $95 \%$ prediction intervals to categorise heterogeneity; instead, we used the $Q$ test $p$ value and the $I^{2}$ results, which might have decreased the accuracy of the GRADE assessment. All the primary studies included in the published meta-analyses were case-control or cross-sectional studies, so the risk of recall bias or selection bias could not be avoided. A large effect size was found in some meta-analyses; all of them were unadjusted for confounders (eg, body mass index, smoking, abdominal obesity, frequent coughing, or frequent use of $\beta 2$ agonists, or Helicobacter infection), which may have affected the validity of the evidence. The small sample size and high heterogeneity of meta-analyses may decrease the strength and validity of evidence. The method of diagnosis of GORD includes clinical history and questionnaires, proton pump inhibitor trial, endoscopy and biopsy, pH-metry and ambulatory reflux monitoring. ${ }^{77}$ GORD is usually diagnosed by symptoms. The latest studies have shown that the specificity and specificity of GORD diagnosis by symptoms are $64.9 \%$ and $71.4 \%$, respectively. ${ }^{78}$ Therefore, the diversity of diagnostic methods for GORD results might also decrease the strength and validity of evidence. Lastly, we included the largest and latest meta-analyses, but these might not have had the highest certainty of evidence and thus might have influenced the conclusions generated by this umbrella review.

Several factors should be considered in future studies to achieve high certainty of evidence. Prospective cohort studies on the associations between GORD and various diseases with a larger sample size should be conducted in the future using time-varying exposure (GORD duration, control or treatment). Adjusting for confounders such as age, sex, smoking status, body mass index, frequent use of $\beta 2$ agonists and $H$. pylori infection may assist in rendering the role of GORD in the development of these diseases much clearer in future. Evidence from meta-analyses with publication bias should be reconfirmed in future studies. Most of the meta-analyses had moderate to high heterogeneity, indicating that associations could be inflated or may be false positives. Well-controlled primary studies should be conducted to reduce the heterogeneity of meta-analyses. The association with high heterogeneity should be further explored by conducting meta-analyses with low heterogeneity. We found gaps in evidence derived from systematic reviews and metaanalyses on the associations between GORD and health disorders in this umbrella review because meta-analyses on those topics have not been performed. However, some individual studies have described these associations despite inconsistent conclusions. ${ }^{45} 79$ Thus, future meta-analyses should be conducted to confirm those findings and resolve gaps in information.

\section{CONCLUSIONS}

This umbrella review systematically summarised the associations between GORD and various diseases derived from systematic reviews and meta-analyses and evaluated their methodological quality of the meta-analyses and the certainty of existing evidence. GORD is associated with oesophageal adenocarcinoma, Barrett's oesophagus, NAFLD, CRS, laryngeal malignancy, OSAHS, IPF and COPD exacerbation, but it is not associated with the risk of pharyngeal cancer. There was high certainty of evidence that GORD is a risk factor of COPD exacerbation and OAC. Further studies with systematic review and meta-analyses of high methodological quality that included prospective cohort studies with large sample sizes and adjusted confounders are needed to confirm these associations.

\section{Author affiliations}

${ }^{1}$ Graduate School, Jiang Xi University of Traditional Chinese Medicine, Nanchang, Jiangxi, China

${ }^{2}$ Department of Administration, The First Affiliated Hospital of Guangxi University of Chinese Medicine, Nanning, Guangxi, China 
${ }^{3}$ Department of Gastroenterology, The First Affiliated Hospital of Guangxi University of Chinese Medicine, Nanning, Guangxi, China

${ }^{4}$ Department of Center of Preventive Disease Treatment, The First Affiliated Hospital of Guangxi University of Chinese Medicine, Nanning, Guangxi, China

Acknowledgements We would like to thank LetPub (www.letpub.com) for its linguistic assistance during the preparation of this manuscript.

Contributors JT wrote the protocol and registered it in PROSPERO. LL and JT screened the articles according to the eligibility and exclusion criteria. $\mathrm{YZ}$ and $\mathrm{XL}$ independently searched the databases and imported the yielded articles to EndNote software. LL and CY extracted the data. DW and CY conducted the AMSTAR 2 eveluating. LL and JT conducted the evidence classifications. XH checked the data and results. JX and RC conduct statistical analyses. LL, JT and CY wrote the draft of the paper, and XH revised the paper. SX was contributed in concept design, guidance and arbitrating all discrepancies and was also responsible for the final content.

Funding This work was funded by National Natural Science Foundation of China (no. 81573914, no.81460723 and no.82004299); the special foundation for science and technology of guangxi administration of traditional Chinese medicine (no. GZZX15-02); the project of improving the evidence-based ability of specialised diseases (gastro-oesophageal reflux disease) in the field of traditional Chinese medicine (TCM) digestion (no. 2019XZZX-XH003).

Competing interests None declared.

Patient consent for publication Not required.

Provenance and peer review Not commissioned; externally peer reviewed.

Data availability statement All data relevant to the study are included in the article or uploaded as supplementary information.

Supplemental material This content has been supplied by the author(s). It has not been vetted by BMJ Publishing Group Limited (BMJ) and may not have been peer-reviewed. Any opinions or recommendations discussed are solely those of the author(s) and are not endorsed by BMJ. BMJ disclaims all liability and responsibility arising from any reliance placed on the content. Where the content includes any translated material, BMJ does not warrant the accuracy and reliability of the translations (including but not limited to local regulations, clinical guidelines, terminology, drug names and drug dosages), and is not responsible for any error and/or omissions arising from translation and adaptation or otherwise.

Open access This is an open access article distributed in accordance with the Creative Commons Attribution Non Commercial (CC BY-NC 4.0) license, which permits others to distribute, remix, adapt, build upon this work non-commercially, and license their derivative works on different terms, provided the original work is properly cited, appropriate credit is given, any changes made indicated, and the use is non-commercial. See: http://creativecommons.org/licenses/by-nc/4.0/.

ORCID iD

Sheng Xie http://orcid.org/0000-0003-4276-3068

\section{REFERENCES}

1 Iwakiri K, Kinoshita Y, Habu Y, et al. Evidence-Based clinical practice guidelines for gastroesophageal reflux disease 2015. J Gastroenterol 2016;51:751-67.

2 Eusebi LH, Ratnakumaran R, Yuan Y, et al. Global prevalence of, and risk factors for, gastro-oesophageal reflux symptoms: a metaanalysis. Gut 2018;67:430-40.

3 Khan HN, Suleman A, Ullah R, et al. Gastro oesophageal reflux diseases in chronic obstructive pulmonary disease patients. J Ayub Med Coll Abbottabad 2018;30:64-6.

4 Chen C-H, Lin C-L, Kao C-H. Association between gastroesophageal reflux disease and coronary heart disease: a nationwide populationbased analysis. Medicine 2016;95:e4089.

5 Huang C-C, Chan W-L, Luo J-C, et al. Gastroesophageal reflux disease and atrial fibrillation: a nationwide population-based study. PLoS One 2012;7:e47575.

6 You Z-H, Perng C-L, Hu L-Y, et al. Risk of psychiatric disorders following gastroesophageal reflux disease: a nationwide populationbased cohort study. Eur J Intern Med 2015;26:534-9.

7 Kim SY, Park B, Lim H, et al. Gastroesophageal reflux disease increases the risk of chronic rhinosinusitis: a nested case-control study using a national sample cohort. Int Forum Allergy Rhinol 2019;9:357-62
8 Anis MM, Razavi M-M, Xiao X, et al. Association of gastroesophageal reflux disease and laryngeal cancer. World J Otorhinolaryngol Head Neck Surg 2018;4:278-81.

9 Aromataris E, Fernandez R, Godfrey CM, et al. Summarizing systematic reviews: methodological development, conduct and reporting of an umbrella review approach. Int J Evid Based Healthc 2015;13:132-40.

10 Poole R, Kennedy OJ, Roderick P, et al. Coffee consumption and health: umbrella review of meta-analyses of multiple health outcomes. BMJ 2018;360:k194.

11 Shamseer L, Moher D, Clarke M, et al. Preferred reporting items for systematic review and meta-analysis protocols (PRISMA-P) 2015: elaboration and explanation. BMJ 2015;350:g7647.

12 Shea BJ, Reeves BC, Wells G, et al. AMSTAR 2: a critical appraisal tool for systematic reviews that include randomised or nonrandomised studies of healthcare interventions, or both. BMJ 2017;358:j4008.

13 Balshem H, Helfand M, Schünemann HJ, et al. Grade guidelines: 3. rating the quality of evidence. J Clin Epidemiol 2011;64:401-6.

14 Guyatt G, Oxman AD, Akl EA, et al. Grade guidelines: 1. IntroductionGRADE evidence profiles and summary of findings tables. J Clin Epidemiol 2011;64:383-94.

15 Dent J, Holloway RH, Eastwood PR. Systematic review: relationships between sleep and gastro-oesophageal reflux. Aliment Pharmacol Ther 2013;38:657-73.

16 Pace F, Pallotta S, Tonini M, et al. Systematic review: gastrooesophageal reflux disease and dental lesions. Aliment Pharmacol Ther 2008;27:1179-86.

17 San Giorgi MRM, Helder HM, Lindeman R-JS, et al. The association between gastroesophageal reflux disease and recurrent respiratory papillomatosis: a systematic review. Laryngoscope 2016;126:2330-9.

18 Picos A, Badea ME, Dumitrascu DL. Dental erosion in gastroesophageal reflux disease. A systematic review. Clujul Med 2018;91:387-90.

19 Firouzei MS, Khazaei S, Afghari P, et al. Gastroesophageal reflux disease and tooth erosion: SEPAHAN systematic review No. 10. Dent Res J 2011;8:S9-14.

20 Smits MJ, van Wijk MP, Langendam MW, et al. Association between gastroesophageal reflux and pathologic apneas in infants: a systematic review. Neurogastroenterol Motil 2014;26:1527-38.

21 Hershcovici T, Jha LK, Johnson T, et al. Systematic review: the relationship between interstitial lung diseases and gastro-oesophageal reflux disease. Aliment Pharmacol Ther 2011;34:1295-305

22 Weaver EM. Association between gastroesophageal reflux and sinusitis, otitis media, and laryngeal malignancy: a systematic review of the evidence. Am J Med 2003;115 Suppl 3A:81-9.

23 Magliulo G, lannella G, Polimeni A, et al. Laryngopharyngeal reflux in obstructive sleep apnoea patients: literature review and metaanalysis. Am J Otolaryngol 2018;39:776-80.

24 Eusebi LH, Ratnakumaran R, Bazzoli F, et al. Prevalence of dyspepsia in individuals with gastroesophageal Reflux-Type symptoms in the community: a systematic review and meta-analysis. Clin Gastroenterol Hepatol 2018;16:39-48.

25 Sun X-M, Tan J-C, Zhu Y, et al. Association between diabetes mellitus and gastroesophageal reflux disease: a meta-analysis. World J Gastroenterol 2015;21:3085

26 Niigaki M, Adachi K, Hirakawa K, et al. Association between metabolic syndrome and prevalence of gastroesophageal reflux disease in a health screening facility in Japan. $J$ Gastroenterol 2013:48:463-72.

27 Corley DA, Kubo A. Body mass index and gastroesophageal reflux disease: a systematic review and meta-analysis. Am J Gastroenterol 2006;101:2619-28.

28 Lovell RM, Ford AC. Prevalence of gastro-esophageal refluxtype symptoms in individuals with irritable bowel syndrome in the community: a meta-analysis. Am J Gastroenterol 2012;107:1793-801

29 Lauriti G, Lisi G, Lelli Chiesa P, et al. Gastroesophageal reflux in children with neurological impairment: a systematic review and metaanalysis. Pediatr Surg Int 2018;34:1139-49.

30 Niu X, Wu Z-H, Xiao X-Y, et al. The relationship between adenoid hypertrophy and gastroesophageal reflux disease: a meta-analysis. Medicine 2018:97:e12540.

31 Wang XT, Zhang M, Chen CY, et al. [Helicobacter pylori eradication and gastroesophageal reflux disease: a Meta-analysis]. Zhonghua Nei Ke Za Zhi 2016;55:710-6.

32 Sakae TM, Pizzichini MMM, Teixeira PJZ, et al. Exacerbations of COPD and symptoms of gastroesophageal reflux: a systematic review and meta-analysis. J Bras Pneumol 2013;39:259-71. 
33 Taylor JB, Rubenstein JH. M1094 Meta-Analysis of the Association of Gastroesophageal Reflux Disease With Barrett's Esophagus: NO Association With Short Segment Barrett's Esophagus. Gastroenterology 2010;138:S330.

34 Taylor JB, Rubenstein JH. T1055 meta-analysis of association of gastroesophageal reflux symptoms and esophageal adenocarcinoma. Gastroenterology 2009;136:A489.

35 Magliulo G, lannella G, Polimeni A, et al. Laryngopharyngeal reflux in obstructive sleep apnoea patients: literature review and metaanalysis. Am J Otolaryngol 2018;39:776-80.

36 Bédard Méthot D, Leblanc Évelyne, Lacasse Y. Meta-Analysis of gastroesophageal reflux disease and idiopathic pulmonary fibrosis. Chest 2019;155:33-43.

37 Havemann BD, Henderson CA, El-Serag HB. The association between gastro-oesophageal reflux disease and asthma: a systematic review. Gut 2007;56:1654-64

38 Huang C, Liu Y, Shi G. A systematic review with meta-analysis of gastroesophageal reflux disease and exacerbations of chronic obstructive pulmonary disease. BMC Pulm Med 2020;20.

39 Leason SR, Barham HP, Oakley G, et al. Association of gastrooesophageal reflux and chronic rhinosinusitis: systematic review and meta-analysis. Rhinology 2017;55:3-16.

40 Parsel SM, Wu EL, Riley CA, et al. Gastroesophageal and Laryngopharyngeal Reflux Associated With Laryngeal Malignancy: A Systematic Review and Meta-analysis. Clin Gastroenterol Hepatol 2019;17:1253-64.

41 Rubenstein JH, Taylor JB. Meta-Analysis: the association of oesophageal adenocarcinoma with symptoms of gastro-oesophageal reflux. Aliment Pharmacol Ther 2010;32:1222-7.

42 Taylor JB, Rubenstein JH. Meta-Analyses of the effect of symptoms of gastroesophageal reflux on the risk of Barrett's esophagus. Am J Gastroenterol 2010;105:1730-7.

43 Wijarnpreecha K, Panjawatanan P, Thongprayoon C, et al. Association between gastroesophageal reflux disease and nonalcoholic fatty liver disease: a meta-analysis. Saudi J Gastroenterol 2017;23:311-7.

44 Wu Z-H, Yang X-P, Niu X, et al. The relationship between obstructive sleep apnea hypopnea syndrome and gastroesophageal reflux disease: a meta-analysis. Sleep Breath 2019;23:389-97.

45 Zhang D, Zhou J, Chen B, et al. Gastroesophageal reflux and carcinoma of larynx or pharynx: a meta-analysis. Acta Otolaryngol 2014;134:982-9.

46 Mirza S, Clay RD, Koslow MA, et al. Copd guidelines: a review of the 2018 gold report. Mayo Clin Proc 2018;93:1488-502.

47 Vogelmeier CF, Criner GJ, Martinez FJ, et al. Global strategy for the diagnosis, management, and prevention of chronic obstructive lung disease 2017 report. gold executive summary. Am J Respir Crit Care Med 2017;195:557-82.

48 Whiteman DC, Appleyard M, Bahin FF, et al. Australian clinical practice guidelines for the diagnosis and management of Barrett's esophagus and early esophageal adenocarcinoma. J Gastroenterol Hepatol 2015;30:804-20.

49 Subspecialty Group of Rhinology, Editorial Board of Chinese Journal of Otorhinolaryngology Head and Neck Surgery, Subspecialty Group of Rhinology, Society of Otorhinolaryngology Head and Neck Surgery, Chinese Medical Association. [Chinese guidelines for diagnosis and treatment of chronic rhinosinusitis (2018)]. Zhonghua Er Bi Yan Hou Tou Jing Wai Ke Za Zhi 2019;54:81-100.

50 Ichinose $M$, Sugiura $\mathrm{H}$, Nagase $\mathrm{H}$, et al. Japanese guidelines for adult asthma 2017. Allergol Int 2017:66:163-89.

51 Al-Moamary MS, Alhaider SA, Alangari AA, et al. The Saudi Initiative for Asthma - 2019 Update: Guidelines for the diagnosis and management of asthma in adults and children. Ann Thorac Med 2019;14:3-48.

52 Lee $\mathrm{SH}, \mathrm{Yeo} \mathrm{Y}, \mathrm{Kim} \mathrm{TH}$, et al. Korean guidelines for diagnosis and management of interstitial lung diseases: Part 2. idiopathic pulmonary fibrosis. Tuberc Respir Dis 2019;82:102-17.

53 Shaheen NJ, Falk GW, lyer PG, et al. Acg clinical guideline: diagnosis and management of Barrett's esophagus. Am J Gastroenterol 2016;111:30-50.

54 Chen Y. Gastroesophageal reflux disease and non-digestive tract diseases. Expert Rev Gastroenterol Hepatol 2015;9:685-92.

55 Houghton LA, Lee AS, Badri $\mathrm{H}$, et al. Respiratory disease and the oesophagus: reflux, reflexes and microaspiration. Nat Rev Gastroenterol Hepatol 2016;13:445-60.
56 Pomari C, Mauroner L, Paiano S, et al. Bronchial reacutization and gastroesophageal reflux: is there a potential clinical correlation? Ann Transl Med 2016;4:304.

57 Starosta V, Griese M. Protein oxidation by chronic pulmonary diseases in children. Pediatr Pulmonol 2006;41:67-73.

58 Iliaz S, Iliaz R, Onur ST, et al. Does gastroesophageal reflux increase chronic obstructive pulmonary disease exacerbations? Respir Med 2016;115:20-5

59 Starosta V, Kitz R, Hartl D, et al. Bronchoalveolar pepsin, bile acids, oxidation, and inflammation in children with gastroesophageal reflux disease. Chest 2007;132:1557-64.

60 Undem BJ, Kollarik M. The role of vagal afferent nerves in chronic obstructive pulmonary disease. Proc Am Thorac Soc 2005;2:355-60.

61 Tan J-J, Wang L, Mo T-T, et al. Pepsin promotes IL-8 signalinginduced epithelial-mesenchymal transition in laryngeal carcinoma. Cancer Cell Int 2019;19:64.

62 Yoshida N. [Cytokine expression in GERD]. Nihon Rinsho 2007;65:813-21.

63 Doukas SG, Cardoso B, Tower JI, et al. Biliary tumorigenic effect on hypopharyngeal cells is significantly enhanced by $\mathrm{pH}$ reduction. Cancer Med 2019;8:4417-27.

64 Bhardwaj V, Gokulan RC, Horvat A, et al. Activation of NADPH oxidases leads to DNA damage in esophageal cells. Sci Rep 2017;7:9956.

65 Perfilyeva YV, Abdolla N, Ostapchuk YO, et al. Chronic inflammation contributes to tumor growth: possible role of L-Selectin-Expressing myeloid-derived suppressor cells (MDSCs). Inflammation 2019;42:276-89.

66 Southwood JE, Hoekzema CR, Samuels TL, et al. The impact of pepsin on human nasal epithelial cells in vitro: a potential mechanism for extraesophageal reflux induced chronic rhinosinusitis. Ann Otol Rhinol Laryngol 2015;124:957-64.

67 loannidis JPA. Why most published research findings are false. PLOS Med 2005;2:e124.

68 loannidis JPA. Why most discovered true associations are inflated. Epidemiology 2008;19:640-8.

69 Dwan K, Gamble C, Williamson PR, et al. Systematic review of the empirical evidence of study publication bias and outcome reporting bias - an updated review. PLoS One 2013;8:e66844.

70 Deng C, Lu Q, Gong B, et al. Stroke and food groups: an overview of systematic reviews and meta-analyses. Public Health Nutr 2018;21:766-76.

71 Jung JH, Dahm P. Reaching for the stars - rating the quality of systematic reviews with the Assessment of Multiple Systematic Reviews (AMSTAR) 2. BJU Int 2018;122:717-8.

72 Shea BJ, Hamel C, Wells GA, et al. AMSTAR is a reliable and valid measurement tool to assess the methodological quality of systematic reviews. J Clin Epidemiol 2009;62:1013-20.

73 Schünemann HJ, Mustafa RA, Brozek J, et al. Grade guidelines: 22. The grade approach for tests and strategies-from test accuracy to patient-important outcomes and recommendations. J Clin Epidemiol 2019;111:69-82.

74 Kallmayer MA, Salvermoser M, Knappich C, et al. Quality appraisal of systematic reviews, and meta-analysis of the hospital/surgeon-linked volume-outcome relationship of carotid revascularization procedures. J Cardiovasc Surg 2019;60:354-63.

75 Sanabria A, Kowalski LP, Nixon I, et al. Methodological quality of systematic reviews of intraoperative neuromonitoring in thyroidectomy: a systematic review. JAMA Otolaryngol Head Neck Surg 2019;145:563-73.

76 Yi M, Wu X, Zhuang W, et al. Tea consumption and health outcomes: umbrella review of meta-analyses of observational studies in humans. Mol Nutr Food Res 2019;63:e1900389:1900389.

77 Cesario S, Scida S, Miraglia C, et al. Diagnosis of GERD in typical and atypical manifestations. Acta Biomed 2018;89:33-9.

78 Gong EJ, Jung KW, Min Y-W, et al. Validation of the Korean version of the gastroesophageal reflux disease questionnaire for the diagnosis of gastroesophageal reflux disease. $J$ Neurogastroenterol Motil 2019;25:91-9.

79 Lin H-C, Xirasagar S, Lee C-Z, et al. The association between gastrooesophageal reflux disease and subsequent rheumatoid arthritis occurrence: a nested case-control study from Taiwan. BMJ Open 2017;7:e016667. 